\title{
Review: Design and Analysis of Nanoscale Bioassemblies
}

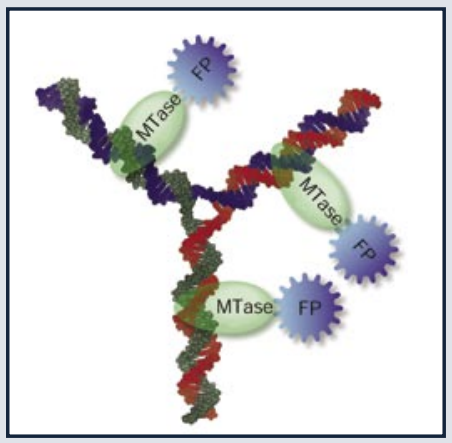

The production of supramolecular assemblies (SMAs) of biological molecules constitutes the foundation of one of the long-term goals of the emerging field of bionanotechnology, construction of nanoscale devices capable of a variety of diverse functions. In this comprehensive review of nanoscale bioassembly, Clark et al. (p. 992) discuss the production, in aqueous media, of SMAs that do not occur in nature. They review the types of structures that have been produced by protein-protein, DNA-DNA, and protein-DNA interactions, describing the detailed organization of the constituent molecules. The authors discuss the range of analytical techniques, from centrifugation to surface plasmon resonance imaging, that are currently employed to characterize SMAs, noting advantages and limitations of each. This review will serve as a valuable compendium on this important area of emerging biotechnology.

\section{Take This to the Bank}

Proper biospecimen collection procedures are critical for the development of diagnostic tools and identification of therapeutic targets. Often, background information about the collected tissue is lacking despite efforts to control experimental variables during tissue processing. Spruessel et al. (p. 1030) analyze the impact of ischemia on gene and protein expression profiles of normal and malignant colon tissue in order to assess the effects of tissue collection time on data quality. The authors conclude that to further improve data mining and to identify artifacts, standardized sample collection procedures should include extensive documentation of all exogenous variables that directly affect the molecular composition of tissues and the subsequent generation of analytical data. Such documentation, together with comprehensive clinical and follow-up data, will significantly improve the success of modern screening technologies used in tissue banking.

\section{In Hot Water No More}

Hyperthermia can be lethal to cancer cells, and thus the mechanism of this process is of considerable interest. In vitro, this phenomenon traditionally has been studied by submerging Parafilm ${ }^{\circledR}$-sealed multiwell plates in hot water baths. Aside from the inconvenience to the user, the cells may suffer from temperature fluctuations during the sealing process and a lack of $\mathrm{CO}_{2}$ while submerged in the water bath. Not surprisingly, Shellman et al. (p. 968) felt there must be a better way. The group's first priority was to develop an accurate means of measuring temperature in individual wells of a 96-well plate. This information would be useful not only in comparing temperature control methods but also in calibrating the appropriate thermal dose for hyperthermia experiments. The resulting device uses fine thermocouples with fast response time to measure temperature to within $0.2^{\circ} \mathrm{C}$. The researchers then used their system to compare heating by water bath, thermal cycler, or incubator. For the incubator case, the group also compared the effects of setting the plates directly on the rack or on a copper block hypothesized to improve heat transfer. The copper block-incubator combination provided the most reproducible results and thus represents a convenient alternative to the hot water bath for hyperthermia experiments.

\section{Structures Without Strictures}

Structural biologists have their work cut out for them. There are some 2.5 million nonredundant protein sequences in GenBank $^{\circledR}$, but only about 24,000 protein structures in the Protein Data Bank (PDB). Clearly, increasing structure determination throughput is a top priority. Improved

bioinformatics will play a big role in accelerating the pace

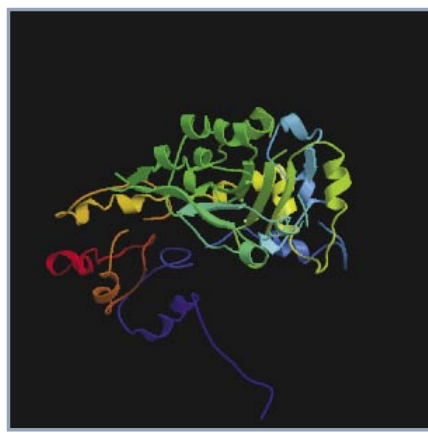
of protein structure discovery, and with this in mind, Canaves et al. (p. 1040) developed a primer selection tool for amplification of full-length open reading frames (ORFs). Appropriately enough, the result is not small-scale. An unlimited number of sequences of any length can be uploaded, and the tool can generate 1000 primer pairs per minute. The program is accessible on the web, and, for those not content with working from afar, the underlying Perl module engine is also freely accessible. Of course, any primer picker is only as good as its amplification success rate. Canaves et al. tested theirs on targets from one eukaryote, four Archaea, and nine bacteria, obtaining success rates of $60 \%$ to $94 \%$. Researchers interested in chipping away

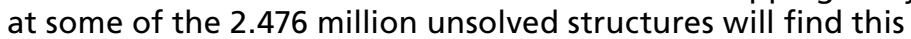
primer selection tool an invaluable first step in their discovery pipeline. 


\section{Cyanobacterial Spectrum}

Modulation of pigment level is an essential adaptation response in many photosynthetic organisms who adjust light harvesting to environmental conditions and thus avoid oxidative damage by excess absorbed light. Cyanobacteria possess a pigment complex, the phycobilisome, which

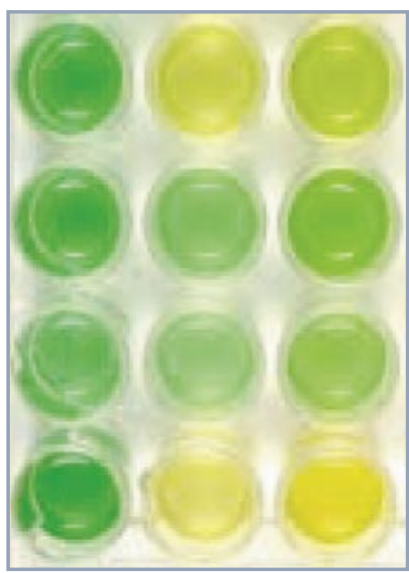
harvests light energy and transfers it to the photosynthetic reaction center under normal conditions. In times of stress (starvation), this complex is degraded. Cyanobacterial mutants have been previously identified, using a visual screen, that are unable to decrease their light-harvesting phycobilisome content in response to nutrient deprivation. Perelman et al. (p. 948) describe the application of fluorescence-activated cell

sorting to isolate Cyanobacterial mutants. Their method has advantages over the visual screen in that it is neither laborious nor time-consuming. The authors demonstrate that their method can also distinguish between partial phenotypes and does not generate a high rate of false positives unlike the visual screening method.

\section{Phage in Frame}

Phage display can involve selection from random peptide sequences or CDNA libraries. The latter approach, however, can be frustrated by the necessity of ensuring that the cDNA is in the same frame as the coding sequence of the phage coat protein. Faix et al. (p. 1018) describe an open reading frame (ORF) selection strategy that they apply to a fragmented CDNA library to weed out clones with frame-shifts and premature stop codons. The strategy involves first directionally cloning the CDNA fragments into a plasmid such that they appear between coding sequences for the plll coat protein and $\beta$-lactamase. A selection step on ampicillin-containing medium ensures that only those clones that allow read-through to the $\beta$-lactamase sequence survive. The selected CDNAs are then cloned into a plll display phagemid for subsequent affinity selection. The researchers show that their procedure results in increased cDNA expression, and they validate the technique by demonstrating that biopanning with a monoclonal antibody against human placental lactogen does indeed select CDNA fragments coding for that protein.

\section{COOL Cloning}

Insertion of short DNA sequences into plasmids is a widely applied technique, but it can be complicated by lack of precision and the need to screen and separate clones in order to identify the insert of interest. Blachinsky et al. (p. 933) describe a straightforward procedure for introducing oligonucleotides into plasmids in the desired number, orientation, and order. The protocol entails the sequential insertion of sequences in separate ligation reactions, each of which restores the original restriction sites of the plasmid. The procedure requires only basic cloning skills, can be used with any combination of restriction enzymes, is applicable to inserts of any length (with some caveats), and, although demonstrated here in yeast, can be used in any system. The authors dub their method "Controlled and Ordered Oligonucleotides Ligations" (COOL).

\section{Double Trouble for Restriction Digests}

Plasmid construction strategies often involve directional ligation, and the difficulties in detecting inefficient double digestion is a major cause of cloning failures. Anand et al. (p. 982) describe the design and construction of plasmids, referred to as monitor plasmids, which can be used to evaluate the efficiency of doubly digested plasmids and PCR products. Four monitor plasmids were constructed, each containing three polylinkers in which 32 popular cloning enzymes are distributed, allowing at least one plasmid in the set to be diagnostic for each enzyme pair. The plasmids also serve as versatile self-monitoring cloning vectors for any popular site combination. This work addresses an important issue in cloning studies and promises to be widely applicable as a tool for plasmid construction. 